\title{
BRANCH-POINT MANIFOLDS ASSOCIATED WITH A LINEAR SYSTEM OF PRIMALS*
}

BY T. R. HOLLCROFT

1. Introduction. Linear $\infty^{\alpha}$ systems of primals in $S_{r}$ have been treated $\dagger$ only for $\alpha=1,2$. The properties of a linear system are obtained from the characteristics of the jacobian and of the branch-point manifold associated with the system. There are, at present, no means for deriving most of the characteristics of a singular primal or manifold in $S_{r}$, especially for $r>4$.

In this paper, a theorem is developed giving a set of characteristics of the branch-point manifolds of the system and its sub-systems. This is a step, not only toward the characterization of a general linear system in $S_{r}$, but also toward the study of singular manifolds which contain both nodal and cuspidal manifolds. $\ddagger$

2. Definitions and Basic Considerations. In $S_{r}$, the linear $\infty^{r}$ system, $F_{r}$, of primals is defined by the equation

$$
\sum \lambda_{i} f_{i}=0, \quad(i=1,2, \cdots, r+1),
$$

in which the $f_{i}$ are general algebraic functions of order $n$ in the $r+1$ homogeneous variables $x_{i}$. Then $f_{i}=0$ is the equation of a primal of order $n$ without singularities in $S_{r}$.

The primals of $F_{r}$ in the $r$-space $(x)$ are in $(1,1)$ correspondence with the primes $\sum a_{i} y_{i}=0,(i=1,2, \cdots, r+1)$, of an $r$ space $(y)$. This correspondence is defined by the equations

$$
\rho y_{i}=f_{i}, \quad(i=1,2, \cdots, r+1) .
$$

* Presented to the Society, September 12, 1935.

$\dagger$ T. R. Hollcroft, Pencils of hypersurfaces, American Journal of Mathematics, vol. 53 (1931), pp. 929-936; Nets of manifolds in i dimensions, Annali di Matematica, (4), vol. 5 (1927-28), pp. 261-267.

$\ddagger$ These terms will be used: node, a double point of a manifold at which the quadric hypercone is entirely general; nodal manifold of a manifold $f$, a manifold for every point of which (except points on pinch and singular loci) the two tangent linear manifolds to $f$ are distinct; cuspidal manifold of $f$, a manifold for all points of which the two tangent linear manifolds to $f$ coincide; cone to mean hypercone for $r>3$. 
To a point $P$ of $(y)$, considered as bearing $\infty^{r-1}$ primes, corresponds $n^{r}$ points of $(x)$. These $n^{r}$ points are the basis points of the $\infty^{r-1}$ linear system of primals $F_{r-1}$ in which the primals are in $(1,1)$ correspondence with the primes in $(y)$ through $P$. Since $(y)$ contains $\infty^{r}$ points, $F_{r}$ contains $\infty^{r}$ linear systems $F_{r-1}$.

In the general case, to an $S_{k}$ of $(y),(k \leqq r-1)$, considered as bearing $\infty^{r-k-1}$ primes, corresponds in $(x)$ the basis manifold $M_{k}$ (of dimension $k$ and order $n^{r-k}$ ) of an $\infty^{r-k-1}$ linear system of primals $F_{r-k-1}$ in which the primals are in $(1,1)$ correspondence with the primes of $(y)$ through $S_{k}$. Since $(y)$ contains $\infty{ }^{(k+1)(r-k)}$ linear manifolds $S_{k}$, the system $F_{r}$ contains $\infty^{(k+1)(r-k)}$ linear systems $F_{r-k-1}$.

The jacobian $J$ of the linear system $F_{r}$ is a primal of order $(r+1)(n-1)$. It is the locus of double points and contacts of primals of $F_{r}$. The jacobian $J$ also contains the jacobian manifolds of all the linear systems of primals contained in $F_{r}$ such that the jacobians of the systems $F_{r-k-1}$ form a $(k+1)(r-k)$ parameter linear system of manifolds on $J$. Likewise $J$ contains the singularities of higher order and contacts of higher order of primals of $F_{r}$ and of all linear systems of primals contained in $F_{r}$. The jacobian $J$ has no actual singularities, only apparent singular manifolds.

The $(1,1)$ correspondence between the primals of $F_{r}$ and the primes of $(y)$ establishes a $\left(1, n^{r}\right)$ involution between the points of $(y)$ and $(x)$, and $J$ is the locus of coincidences of this involution. The image of $J$ in $(y)$ is the branch-point primal $L$, the locus of points such that all primals of each associated $F_{r-1}$ have contact with a line at a point on $J$. The $\infty^{r-1}$ contacts generate $J$.

$L$ is also the envelope of primes of $(y)$ which correspond to primals of $F_{r}$ that have a node. To the points of contact of primes with $L$ correspond uniquely the nodes, which lie on $J$.

The order $\mu_{0}$ of $L$ is the number of points in which $J$ and $r-1$ primals of $F_{r}$ intersect, that is, $\mu_{0}=(r+1)(n-1) n^{r-1}$.

The classes of $L$ are defined as follows:

$\mu_{1}$, the order of the tangent cone to $L$ from a point;

$\mu_{2}$, the order of the tangent cone to $L$ from a line;

$\mu_{k+1}$, the order of the tangent cone to $L$ from an $S_{k}$;

$\mu_{r-1}$, the number of tangent primes to $L$ from an $S_{r-2}$. 
3. A Theorem Defining Branch-Point Manifolds of the $F_{r-k-1}$. The primals of an $F_{r-k-1}$ of $F_{r}$ of $(x)$ are in $(1,1)$ correspondence with the primes of $S_{r-k-1}$, a sub-space of $(y)$. This establishes a $(1,1)$ correspondence between the points of $S_{r-k-1}$ and the basis manifolds $M_{k-1}$ of the $(r-k-2)$-parameter linear systems of primals contained in $F_{r-k-1}$. The locus of points of $S_{r-k-1}$ for which all of the primals of the associated $(r-k-2)$-parameter linear systems have contact at one point with a line is the branch-point manifold $L_{r-k-2}$ (primal of $S_{r-k-1}$ ) and the locus of contacts in $(x)$ is the jacobian manifold $J_{r-k-2}$.

As shown in $\$ 2$, the primals of an $(r-k-1)$-parameter linear system of primals belonging to $F_{r}$ in $(x)$ are in $(1,1)$ correspondence with the primes of $(y)$ through an $S_{k}$. The $(k+1)$ st class of $L, \mu_{k+1}$, is the order of the tangent cone enveloped by primes through $S_{k}$ tangent to $L$. To each such tangent prime corresponds a primal of $F_{r-k-1}$ and of $F_{r}$ with a node.

Consider any given $S_{r-k-1}$ of $(y)$. $S_{r-k-1}$ intersects each of the primes through $S_{k}$ in an $S_{r-k-2}$, which is a prime of $S_{r-k-1}$. The primals of $F_{r-k-1}$ are in $(1,1)$ correspondence with these primes $\left[S_{r-k-2}\right.$ of $\left.(y)\right]$ of $S_{r-k-1}$.

Since the order of the tangent cone to $L$ from $S_{k}$ is $\mu_{k+1}$, the section of this tangent cone by $S_{r-k-1}$ is a manifold $V_{r-k-2}$ of dimension $r-k-2$ and order $\mu_{k+1}$. This manifold $V_{r-k-2}$ is the envelope of the primes of $S_{r-k-1}$ which are sections by $S_{r-k-1}$ of the primes of $(y)$ through $S_{k}$ tangent to $L$. Therefore the primes in $S_{r-k-1}$ enveloping $V_{r-k-2}$ are in $(1,1)$ correspondence with the primals of $F_{r-k-1}$ which have a node. But, as previously shown, the $(1,1)$ correspondence between the primals of $F_{r-k-1}$ and the primes of $S_{r-k-1}$ establish an involution in which the branchpoint manifold $L_{r-k-2}$ of $S_{r-k-1}$ is defined as the envelope of primes of $S_{r-k-1}$ which correspond uniquely to primals of $F_{r-k-1}$ that have a node. Therefore, in $S_{r-k-1}$,

$$
L_{r-k-2} \equiv V_{r-k-2} \text {. }
$$

This identity establishes the following theorem.*

The section by an $S_{r-k-1}$ of the tangent cone from an $S_{k}$ to $L$, where $L$ is the branch-point primal in the r-space $(y)$ associated

* This theorem has been established for three dimensions. See T. R. Hollcroft, The general web of algebraic surfaces of order $n$ and the involution defined by it, Transactions of this Society, vol. 35 (1933), p. 859. 
with an $r$-parameter linear system of primals $F_{r}$ of an $r$-space $(x)$, is the branch-point manifold $L_{r-k-2}$ of $S_{r-k-1}$ associated with a linear $(r-k-1)$-parameter system of primals $F_{r-k-1}$ belonging to $F_{r}$.

The order $\mu_{k+1}$ of $L_{r-k-2}$ is also the order of the contour manifolds on $L$ of the tangent cones from an $S_{k}$. These contour manifolds, of dimension $r-k-2$, form a linear system on $L$ and are the respective images of the jacobian manifolds of the $F_{r-k-1}$ contained in $F_{r}$. These jacobian manifolds form a linear system on $J$ of the same respective dimension as the associated linear system of contour manifolds on $L$. Its contour manifold, $L_{r-k-2}$, and its associated jacobian manifold are all in $(1,1)$ correspondence.

4. Relations Resulting from the Theorem. By the above theorem, the $(k+1)$ st class $\mu_{k+1}$ of $L$ is the order of the branch-point manifold $L_{r-k-2}$ associated with an $F_{r-k-1}$ belonging to $F_{r}$.

In the $\left(1, n^{r-k-1}\right)$ involution associated with $F_{r-k-1}$, the condition for a point to lie on $L_{r-k-2}$ is that the primals of $F_{r-k-1}$ have a common tangent $S_{k+2}$ at a common point. The condition that $r-k-1$ primals have a common tangent $S_{k+2}$ at a common point is the tact-invariant of this system of primals. The order of this tact-invariant is*

$$
\mu_{k+1}=\frac{1}{(k+2) !}(r+1) r(r-1)(r-2) \cdots(r-k)(n-1)^{k+2} n^{r-k-2} .
$$

This is, therefore, the order of $L_{r-k-2}$ and the value of $\mu_{k+1}$, the $(k+1)$ st class of $L$.

The order $\mu_{0}$ of $L$ results from the above formula for $k=-1$, that is, the order $\mu_{0}$ is the tact-invariant of $r$ primals of $F_{r}$. The final class of $L, \mu_{r-1}=(r+1)(n-1)^{r}$, is the order of the discriminant of a primal of $F_{r}$ and is not a tact-invariant, since it involves only one primal. The value of $\mu_{r-1}$, however, is also given by the above formula for $k=r-2$.

The class $\mu_{r-2}$ of $L$ is the order of the tangent cone to $L$ from an $S_{r-3}$. This is also the order of the branch-point curve $L_{1}$ associated with a net of primals of $F_{r}$. The complete set of charac-

* T. R. Hollcroft, Tact-invariants of primals in $S_{r}$, Journal of the London Mathematical Society, vol. 11 (1936), p. 24. 
teristics of $L_{1}$ is given in a former paper.* These are also the characteristics of a tangent cone (surface) to $L$ from an $S_{r-3}$. The characteristics of $L_{2}$ and therefore of the tangent cone to $L$ from an $S_{r-4}$ have been found $\dagger$ for $n=2$, but not for a general $n$.

Since the final class $\ddagger$ of a section of $L$ made by an $S_{k+2}$ is $\mu_{k+1}$, the above value of $\mu_{k+1}$ gives the final classes of all sections of $L$ by a linear manifold as well as the orders of all tangent cones to $L$ from a linear manifold. The order of the section of $L$ by any linear manifold is $\mu_{0}$.

In general, $L$ in $(y)$ has both a nodal and a cuspidal manifold, each of dimension $r-2$, and these manifolds are themselves singular. For a linear system of dimension $r$ in $S_{r-1}$, however, $L$ has only a nodal manifold of dimension $r-2$, containing a pinch manifold of dimension $r-3$.

\section{Wells College}

* T. R. Hollcroft, Nets of manifolds in i dimensions, loc. cit.

$\dagger \mathrm{T}$. R. Hollcroft, The web of quadric hypersurfaces in $r$ dimensions, this Bulletin, vol. 41 (1935), pp. 97-103.

$\ddagger$ By final class of an $S_{k+2}$ section of $L$ is meant the number of $S_{k-1}$ through an arbitrary $S_{k}$ (all in $S_{k+2}$ ) tangent to $L$. 